\title{
Impact of Educational Program Regarding Ventilator-Associated Pneumonia Bundle on Critical Care Nurses' Knowledge and Practices
}

\author{
Nahla S. khalil, Hala A. Mohamed, Ola A. Sayed \\ 1-Assistant Prof. of critical care nursing, Cairo university \\ 2-lecturer of critical care nursing and emergency \\ 3-B.S Faculty of nursing, Mansoura university
}

\begin{abstract}
Ventilator-associated pneumonia (VAP) is considered as a leading cause of infection related mortality and morbidity in intensive care units (ICUs) together with increasing the length of ICU stay and raising the cost of antibiotic treatment of VAP especially in developing countries. Education of ICU healthcare providers is widely viewed as a fundamental measure in reducing VAP. So, this study aimed to assess the effectiveness of educational program onknowledge regarding care bundle on prevention of ventilatorassociated pneumonia among intensive care unit nurses.Ventilator-associated pneumonia (VAP) is one of the most common hospital-acquired infections. It is a form of nosocomial pneumonia that occurs in patients undergoing mechanical ventilation for longer than 48 hours. Recently reported VAP rates range from 1 to 4 cases per 1000 ventilator days in industrialized countries and up to 13 cases per 1000 ventilator days in developing countries. VAP is the most serious health care-associated infection and is the leading cause of morbidity and mortality from device-associated infections, especially in the intensive care unit (ICU). Attributable mortality rate estimates vary from $10 \%$ to $65 \%$, with a mean of about 30\%.2 Furthermore, VAP has long been recognized as a cause of prolonged ICU stays and increased hospital costs. An increase of 7 to 9 days in ICU stay has been reported for a patient with VAP, and Kollef et al5 reported in 2012 that the cost per patient increases by US\$40 000 when the patient has VAP. Study design: Quasi-experimental research design was adopted in this study. Setting: the study was conducted in two intensive care units at Meniet ELNasr Central Hospital and Dekirnis Central Hospital. Sample: A convenience sample of all critical care nurses (100) assigned to two groups (50 for the study group, and 50 for control group). Tools:Utilizing one tool, self-administrated questionnaire sheet in Arabic language. It was composed of two parts (demographic data and knowledge assessment questionnaires).Results: the present study results indicated that, the nurses' knowledge of care bundle and ventilator-associated pneumonia was statistically significant improved after the implementation of the educational program either immediately or 3 months later than before its implementation in the study group. While, there is was insignificant differences in the control group throughout the study stages. Conclusion: the study concluded that the health education program was effective in improving nurses' knowledge in the two post-program tests than initial survey in the study group. Recommendation: the study recommended that the provision of continuing education programs is suggested in order to refresh and update nurses' knowledge, as well as reinforce proper practice related to implementation of care bundle and consequently reducing VAP rate.
\end{abstract}

Keywords: Ventilator-associated pneumonia (VAP), Intensive Care Unit, education, care bundle, ICU nurses. 


\section{Introduction:}

Ventilator-associated pneumonia (VAP) is one of the most common encountered hospital-acquired infections seen in the critical care setting. Pneumonia is the second most common nosocomial infection in critically ill patients, affecting $27 \%$ of all critically ill patients (Chithra, \& Raju, 2017). It is the most substantial sub-type of pneumonia, occurring in patients who are mechanically ventilated via an artificial airway, causing substantial morbidity, a two-fold increase in mortality rate, extra costs, all coupled with the prolonged use of ventilators and extended stays in intensive care units (ICU) and hospitals (Mishra, \& Rani, 2020).

The estimated risk of developing VAP is $1.5 \%$ per day and decreases to less than $0.5 \%$ per day after the 14 th day of mechanical ventilation. VAP increases the duration of hospitalization by seven days and thereby the cost of health-care (Timsit, Esaied, Neuville, Bouadma,\& Mourvllier, 2017). VAP is classified into early-onset which occurring less than five days and late-onset occurring equal and more than five days. Furthermore, Bouadma, et al., (2015), cited VAP as "oropharyngeal colonization of pathogens acquired through an exogenous source like infected hands and attire of health care professionals, contaminated respiratory instruments, and intensive care environment, through hospital water or air". These pathogens reach the lung parenchyma and alter the host defense mechanism. Accordingly, Mishra and Rani (2020) pointed out the infection control department role as an indispensable role in the education of the nursing staff on infection control procedures, surveillance nurses on the prevention of VAP among intubated patients. Reduction in the incidence rate of VAP, ultimately decreases the associated burden of illness.

Basically, the Institute for Healthcare Improvement (IHI) recommended the practice of bundles, simple sets of evidence-based practices that, when performed collectively and reliably, have been proven to improve patient outcomes. The components of Ventilator Bundle include: i) Elevating head of the bed to 30-45 degree, ii) Daily 'sedation vacation' to assess readiness to extubate, iii) Peptic ulcer disease prophylaxis, iv) Deep venous thrombosis prophylaxis (Institute for Healthcare Improvement, 2020; Lavallée, Gray, Dumville, Russell, \& Cullum, 2017).

Knowledgeable and skilled nurses are crucial in providing patient care, timely and correct decision minimizes the risks to patients. According to previous international surveys, the theoretical and applied knowledge of critical care nurses has been limited and inadequate regarding evidence based guidelines, protocols and care bundles (Chithra, \& Raju, 2017; Dipali,\& Dumbre,2019).

However, Intensive care nurses are in the best position to bring the evidence based guidelines into practice as they are at the patient's bedside 24 hours a day and therefore they play a substantial role in the prevention of VAP. Nevertheless nurses need to be have aware and up-dated of the problem as well as knowledge on ventilator care bundle so as to adhere to such practices (Arumugam, Sivakumar, \& Balasubramanian, 2016).

Up to the researchers' knowledge, no interventional study was conducted in el Dakahlia governorate, Egypt to improve ICU nurses' knowledge towards 
ventilator

associated

pneumonia.Therefore, this study aimed to assess the effectiveness of educational program onknowledge regarding care bundle on prevention of ventilatorassociated pneumonia among intensive care unit nurses.

Significance of the study: VAP constitutes a serious burden for nurses system and worsens the patient's outcomes; thus, several preventive measures have been implemented. This study reviews the current knowledge on VAP and its preventive measures. From the researcher experience in ICU found unsatisfactory nurses' knowledge and inadequate practices regarding implementation of care bundle for mechanical ventilated patients, which elevating VAP rate.In consequences, education is widely viewed as a necessity in reducing VAP

\section{Aim of the study:}

The study aimed to evaluate the effectiveness of educational program on knowledge regarding care bundle on prevention of ventilator-associated pneumonia among intensive care unit nurses.

\section{Research Hypotheses:}

To fulfill the aim of the study, the following research hypotheses were formulated:

There will be significant difference between post-educational program nurse's knowledge scores than the pre-educational program nurse's knowledge scores in the study group.

There will be insignificant difference in nurse's knowledge scores throughout three study stages in the control group.

\section{Subjects \& Methods}

Research design: Quasiexperimental research design had been used in this study pre and post tested for study and control groups.

Study sample: A convenience sample including all available nurses (100) nurses divided into (50) nurses at Meniet ELNasr intensive care unit and (50) nurses at Dekirnis ICU and willing to participate in the study.

Setting:this study was conducted in two intensive care units at Meniet EL_Nasr Central Hospital and Dekirnis Central Hospital. Each ICU of Meniet El-Nasr and Dekirnis hospital has four rooms, which are divided as: two intensive care; and two-cardiac care. Each ICU unit involves three mechanical ventilator and 50 nurses.

Tools of data collection:

One tool was developed by the researcher to collect the necessary data for this study, and composed of two parts as the following:

Part1:Nurses' demographic and occupational data:It incorporated critical care nurses' demographic data (age, sex, and qualifications), their occupational profile (years of experience) and their training needs (attendance of educational courses regarding critically ill patients' care).

\section{Part2:Self-administrated}

nurses' knowledge regarding VAP bundleQuestionnaire:This uestionnaire was developed and translated to simple Arabic language to investigate critical care nurses' knowledge regarding ventilator associated pneumonia such as definition, signs \& symptoms, diagnostic procedure, VAP bundle and standard precautions. This tool was designed on the highlight of related literatures (Aferu, 2016; Alcan, etal., 2016; Ali, 2013; Al-Sayaghi, 2014; Subramanian, Choy, Gobal, Manso, \& Ng, 2013). These knowledge questions were classified into 13 categories, all of these 
categories involved 101questions (67 multiple choice (MCQ), and 34 Yes and No questions). It was arranged as the following: Respiratory system (6 items); Function of the mechanical ventilator (6 items); Ventilator associated pneumonia (6 items); Mechanical ventilator (5 items); The rendered nursing care for critically ill patients(6 items); The rendered nursing care for endotracheal tube (25 items); The rendered nursing care during Endotracheal tube (ETT) suctioning (14 items); Blood gases analysis(5 items); Nasogastric feeding(5 items); Airway care (2 items); the patient mobilization (3 items); The chest physiotherapy (10 items); The mechanical ventilator circuits sterilization (8 items).

Scoring system of the nurses' knowledge questionnaire:The total score for knowledge was 101 grades. The score ranged from 0 to 101 . Each knowledge question was scored as (1) for the correct answer and (0) for the wrong answer and don't know. A higher score indicated greater nurses' knowledge. Knowledge score was categorized as follows:Scores less than $75 \%$ (less than 75.75 grade) was considered unsatisfactory knowledge level and the scores equal or more than $75 \%$ (equal or more than 75.75 grade) considered satisfactory level of knowledge (Sobeih, et al., 2018; Haza'a, Al-Qubati, Mohammed, Abdel-Aziz,\& Mehany, 2020).

Tools validity: The content validity of the developed tools was tested by five experts of emergency nursing, and their recommended substantial adjustments had been made.

Pilot study: Face validity of the developed tools was tested by conducting a pilot study on the targeted group: $10 \%$ (10 nurses) of the total sample size of critical care nurses, who were selected randomly from the above-mentioned settings. The nurses included in the pilot study were excluded from the whole study sample. Accordingly, the necessary modification was made, some questions were added, and others were clarified or omitted.

Reliability assessment: Internal consistency was tested by using Cronbach's $\alpha$. thatwas emerged as very $\operatorname{good}(0.81)$.

Ethical considerations: Before conducting the study, ethical approval was obtained from the research ethics committee of the faculty of Nursing, Mansoura University. An explanation regarding the aim of the study, and the expected outcomes were clarified for the studied sample. Each nurse was interviewed individually after taken their consent to contribute in the study; they were enhanced cooperatively to participate in the study. Participants were also informed about their right to withdraw from the study at any time without giving a reason. All data and information obtained were protected through coding to ensure confidentiality.

The study procedure: The current study was conducted on four phases: preparatory / or assessment phases, planning phase, implementation phase, and evaluation phase. Data were collected across two study phases that were: preparatory / or assessment phases (baseline data); and evaluation phase (immediate post and follow up data).

Phase1: reparatory/Assessment phase: This phase involved the preparation of data collection tools based on reviewing the pertinent current, national and international literature. Obtaining ethical approval and hospital permission. Verbal informed consents were obtained also from nurses. 
Initial data collection: Baseline assessment was conducted for both study and control group by distributing selfadministered demographic and occupational questionnaire using Tool I (part1), and knowledge assessment test regarding prevention of VAP by Tool 1 (part 2),

\section{Phase2:Planning phase of the educational program \\ - The researcher designed the ventilator-associated pneumonia bundle educational program based on review of related literatures: Kao et al., (2019); Walimanna Gamage (2019); Branco et al., (2020); Alsoda et al., (2019); Álvarez Lerma et al., (2014); Mogyoródi et al.,(2016).}

- The targeted educational program involved 6 group based sessions. These sessions focused on Respiratory system (structure, physiology, and functions); mechanical ventilator (appropriate functions, its alarms, appropriate role of nurse); Ventilator associated pneumonia (definition, causes, Risk factors, and prevention); VAP bundle components; the rendered nursing care for critically ill patients. The developed educational program was tested for content related validity by five critical care and emergency nursing experts.

- The printed booklet containing the content of the program was designed and developed using appropriate illustrations, simple Arabic language, attractive and suitable format

Phase 3: Implementationof the educational program:

- The current study was carried out over period of 18 months, starting from first week of February (2018) up to the first week of July (2019) for data collection and implementation of the educational program. The researcher visited the pervious mentioned study settings, three days/week from 10.00 am to $4.00 \mathrm{pm}$.

- The implementation of the educational program were premeditated based on the actual nurses' needs assessment of the studied nurses through the initial collected data and the reviewed literature. The implementation of the program covered the theoretical aspects concerned to prevention of ventilator-associated pneumonia. It was aimed to improve the nurses' knowledge regarding prevention of VAP. Nurses were divided into small groups, the implementing educational program were proceed over 6 demonstrated sessions (around 45-60 minutes for each) at various morning shifts by moderate of 3 sessions/week. It was given over a period of 18 weeks.

- The researchers utilized different teaching methods during implementation of educational program, it was included: lectures, brain storing, group discussion, clinical scenarios; besides various teaching media such as watching videos, power point presentation and hand-out for prevention of VAP.

Phase 3:Evaluation phase: Immediately after ended the implementation of educational program, nurses' knowledge were evaluated for both the study and control groups using the same study tool of pre-test, for the post-tests (immediately post-test and 3 months follow-up test) that done for comparing between the both study groups to evaluate the program effectiveness. After 
that, the study group nurses at the ICU from Meniet EL_Nasr Hospital, had copies from the booklets of the implemented educational program regarding prevention of VAP.

\section{Statistical}

Analysis:The collected data were coded and entered to the statistical package of social sciences (SPSS) version 24. After complete entry, data were explored for detecting any error, then, it was analyzed by the same program for presenting frequency tables with percentages. Qualitative data was presented as number and percent.

\section{Results:}

Table (1): Critical care nurses' demographic and occupational characteristics:

\begin{tabular}{|c|c|c|c|c|c|c|c|}
\hline \multirow{3}{*}{$\begin{array}{c}\text { Demographic and occupational } \\
\text { characteristics }\end{array}$} & \multicolumn{7}{|c|}{ Total number of nurses $=100$} \\
\hline & \multicolumn{2}{|c|}{$\begin{array}{c}\text { Study } \\
\text { (N=(50 }\end{array}$} & \multicolumn{2}{|c|}{$\begin{array}{c}\text { Control } \\
(\mathrm{N}=\mathbf{5 0}\end{array}$} & \multicolumn{2}{|c|}{$\begin{array}{c}\text { Total } \\
(\mathrm{N}=(\mathbf{1 0 0} \\
\end{array}$} & \multirow[t]{2}{*}{ P-value } \\
\hline & .No & $\%$ & .No & $\%$ & .No & $\%$ & \\
\hline & & & & & & & Age in years \\
\hline $20-29$ & 16 & 32 & 15 & 30 & 31 & 31 & \multirow{5}{*}{$\begin{array}{c}0.51 \\
\& \\
0.775\end{array}$} \\
\hline $30-39$ & 24 & 48 & 22 & 44 & 46 & 46 & \\
\hline $40-50$ & 10 & 20 & 13 & 26 & 23 & 23 & \\
\hline$\geq 50 \mathrm{ys}$ & ---- & ---- & --- & ---- & --- & ---- & \\
\hline SD \pm Mean & \multicolumn{2}{|c|}{$0 . r M \pm r r . r \Lambda$} & \multicolumn{2}{|c|}{$34.12 \pm 5.54$} & \multicolumn{2}{|c|}{$33.70 \pm 5.43$} & \\
\hline \multicolumn{8}{|l|}{ Educational level } \\
\hline Diploma of nursing & 7 & 14 & 12 & 24 & 19 & 19 & \multirow{4}{*}{$\begin{array}{c}2.47 \\
\& \\
0.29\end{array}$} \\
\hline Nursing diploma & 27 & 54 & 20 & 40 & 47 & 47 & \\
\hline Technical Institute & 16 & 32 & 18 & 36 & 34 & 34 & \\
\hline Bachelor of nursing & ---- & ---- & ---- & ----- & ---- & ---- & \\
\hline \multicolumn{8}{|l|}{ Experience years } \\
\hline $5-<10$ & 42 & 84 & 34 & 68 & 76 & 76 & \multirow{3}{*}{$\begin{array}{c}3.5 \\
\& \\
0.06\end{array}$} \\
\hline$\geq 10 \mathrm{ys}$ & 8 & 16 & 16 & 32 & 24 & 24 & \\
\hline $\mathrm{SD} \pm$ Mean & $8.10 \pm 2$ & & $9.34 \pm$ & & $8.72 \pm$ & & \\
\hline \multicolumn{8}{|c|}{ Previous attendance of training program about (VAP) bundle } \\
\hline Yes & 5 & 10 & 11 & 22 & 16 & 16 & \multirow{2}{*}{$\begin{array}{l}r . T V \\
.1 . r \&\end{array}$} \\
\hline No & 45 & 90 & 39 & 78 & 84 & 84 & \\
\hline \multicolumn{8}{|c|}{ Average of cases taken nursing care per shift } \\
\hline 2 cases & 22 & 44 & 24 & 48 & 46 & 46 & \multirow{3}{*}{$\begin{aligned} & \because 17 \\
\ddots & 111 \&\end{aligned}$} \\
\hline 3 cases & 28 & 56 & 26 & 52 & 54 & 54 & \\
\hline SD \pm Mean & \multicolumn{2}{|c|}{$.0 . \pm r .07$} & \multicolumn{2}{|c|}{$2.52 \pm 0.50$} & \multicolumn{2}{|c|}{$2.54 \pm 0.50$} & \\
\hline $\begin{array}{l}\text { There were no } \\
\text { differences amongst the } \\
\text { demographic and oc } \\
\text { characteristics between the gro } \\
\text { indicate the homogeneity of } \\
\text { groups as shown in table (1). } \\
\text { age of the participants in the }\end{array}$ & $\begin{array}{l}\text { significa } \\
\text { baseli } \\
\text { cupation } \\
\text { ups whi } \\
\text { the bo } \\
\text { The me } \\
\text { udy gro }\end{array}$ & & $\begin{array}{l}\text { was } \\
\text { age } \\
\text { grou } \\
\text { the s } \\
\text { grou } \\
\text { Appr } \\
\text { the }\end{array}$ & $\begin{array}{l}.28( \\
\text { S } 34 . \\
\text { Sligh } \\
\text { dy gr } \\
\left(40^{\circ}\right. \\
\text { rimate } \\
\text { rticip. }\end{array}$ & $\begin{array}{l}=5.3 \\
2(\mathrm{SD} \\
\text { y half } \\
\text { up }(5 \\
\text { we } \\
\text { y mor } \\
\text { its in }\end{array}$ & $\begin{array}{l}\text { wher } \\
\text { 54) } \\
\text { the p } \\
\text { ), an } \\
\text { nurs } \\
\text { than } \\
\text { he st }\end{array}$ & $\begin{array}{l}\text { Is the mean } \\
\text { the control } \\
\text { ticipants in } \\
\text { the control } \\
\text { g diploma. } \\
\text { wo-third of } \\
\text { ly and the }\end{array}$ \\
\hline
\end{tabular}


Impact of Educational Program Regarding etc...

control groups had five to less than ten years of experience $(84 \%, 68 \%$, respectively). More than three fourth of the participants in both groups didn't attend any training program about (VAP) bundle $\quad(90 \%, \quad 78 \%$ respectively). Regarding Average of cases taken nursing care per shift, more than half of the participants in both groups take care of three cases per shift.

Table(2):Comparison between study and control group according to the total knowledge leveltoward ventilator associated pneumonia (VAP) bundle pre, immediate, 3 months post program :

\begin{tabular}{|c|c|c|c|c|c|c|c|c|c|c|c|c|c|c|c|c|c|}
\hline \multirow{4}{*}{$\begin{array}{c}\text { Knowledge } \\
\text { level }\end{array}$} & \multicolumn{17}{|c|}{ Nurses No $=100$} \\
\hline & \multicolumn{6}{|c|}{$\begin{array}{c}\text { Study group } \\
(\mathbf{N}=50)\end{array}$} & \multirow{3}{*}{$\begin{array}{c}\text { Test of } \\
\text { significa } \\
\text { nce } \\
\chi^{2} \\
\mathbf{P}\end{array}$} & \multicolumn{6}{|c|}{$\begin{array}{c}\text { Control group } \\
(\mathrm{N}=50)\end{array}$} & \multirow{3}{*}{$\begin{array}{c}\text { Test of } \\
\text { significa } \\
\text { nce } \\
\chi^{2} \\
\mathbf{P}\end{array}$} & \multicolumn{3}{|c|}{ (Study vs Control group) } \\
\hline & \multicolumn{2}{|c|}{ Pre } & \multicolumn{2}{|c|}{$\begin{array}{c}\text { Immedia } \\
\text { te Post }\end{array}$} & \multicolumn{2}{|c|}{$\begin{array}{l}3 \text { months } \\
\text { post }\end{array}$} & & \multicolumn{2}{|c|}{ Pre } & \multicolumn{2}{|c|}{$\begin{array}{c}\text { Immediat } \\
\text { e Post }\end{array}$} & \multicolumn{2}{|c|}{$\begin{array}{c}3 \text { months } \\
\text { post }\end{array}$} & & \multirow{2}{*}{ Pre } & \multirow{2}{*}{$\begin{array}{c}\text { Immedi } \\
\text { ate } \\
\text { post }\end{array}$} & \multirow{2}{*}{$\begin{array}{c}3 \\
\text { month } \\
\text { s post }\end{array}$} \\
\hline & $\begin{array}{l}\mathrm{N} \\
\mathrm{o}\end{array}$ & $\%$ & $\begin{array}{l}\mathrm{N} \\
\mathrm{o}\end{array}$ & $\%$ & $\begin{array}{l}\mathrm{N} \\
\mathrm{o}\end{array}$ & $\%$ & & $\begin{array}{l}\mathrm{N} \\
\mathrm{o}\end{array}$ & $\%$ & $\begin{array}{l}\mathrm{N} \\
\mathrm{o}\end{array}$ & $\%$ & $\begin{array}{l}\mathrm{N} \\
\mathrm{o}\end{array}$ & $\%$ & & & & \\
\hline \multicolumn{18}{|c|}{ Respiratory system } \\
\hline $\begin{array}{l}\text { Unsatisf } \\
\text { actory } \\
\text { knowled } \\
\text { ge }\end{array}$ & 29 & 78 & 5 & $\begin{array}{l}1 \\
0\end{array}$ & 8 & $\begin{array}{l}1 \\
6\end{array}$ & \multirow{3}{*}{$\begin{array}{c}\chi^{2}= \\
50.00, \\
\mathrm{p} \leq 0.001\end{array}$} & 43 & 86 & 44 & 88 & 41 & 82 & \multirow{3}{*}{$\begin{array}{c}\chi^{2}= \\
0.633, \\
\mathrm{p} \leq 0.729\end{array}$} & \multirow{3}{*}{$\begin{array}{c}\chi^{2}= \\
0.328 \\
\mathrm{p} \leq 0.0 . \\
743\end{array}$} & \multirow{3}{*}{$\begin{array}{c}\chi^{2}= \\
7.25, \\
\mathrm{p} \leq 0.001\end{array}$} & \multirow{3}{*}{$\begin{array}{c}x^{2}= \\
6.24 \\
p \leq 0.0 . \\
001\end{array}$} \\
\hline $\begin{array}{l}\begin{array}{l}\text { Satisfactory } \\
\text { knowledge }\end{array} \\
\end{array}$ & 11 & 22 & 95 & $\begin{array}{l}9 \\
0 \\
\end{array}$ & 42 & $\begin{array}{l}8 \\
4 \\
\end{array}$ & & 7 & 14 & 6 & 12 & 9 & 18 & & & & \\
\hline Median & \multicolumn{2}{|c|}{3} & \multicolumn{2}{|c|}{6} & \multicolumn{2}{|c|}{5} & & \multicolumn{2}{|c|}{3} & \multicolumn{2}{|c|}{3} & \multicolumn{2}{|c|}{3} & & & & \\
\hline Function of $\mathbf{n}$ & anica & ventil & & & & & & & & & & & & & & & \\
\hline $\begin{array}{l}\text { Unsatisf } \\
\text { actory } \\
\text { knowled } \\
\text { ge }\end{array}$ & 50 & $\begin{array}{c}10 \\
0\end{array}$ & 25 & $\begin{array}{l}5 \\
0\end{array}$ & 28 & $\begin{array}{l}5 \\
6\end{array}$ & $\chi^{2}=$ & 50 & $\begin{array}{c}10 \\
0\end{array}$ & 50 & $\begin{array}{c}10 \\
0\end{array}$ & 50 & $\begin{array}{c}10 \\
0\end{array}$ & $\chi^{2}=2.34$ & $\begin{array}{c}x^{2}=0.5 \\
83\end{array}$ & $\chi^{2}=8.04$ & $\begin{array}{c}x_{2}=8.0 \\
3,\end{array}$ \\
\hline $\begin{array}{l}\text { Satisfactory } \\
\text { knowledge }\end{array}$ & --- & - & 25 & $\begin{array}{l}5 \\
0 \\
\end{array}$ & 22 & $\begin{array}{l}4 \\
4 \\
\end{array}$ & $\mathrm{p} \leq 0.001$ & -- & -- & -- & -- & $\begin{array}{l}--- \\
- \\
\end{array}$ & - & $\mathrm{p} \leq 0.10$ & $\begin{array}{c}\mathrm{p} \leq 0.56 \\
0\end{array}$ & $\mathrm{p} \leq 0.001$ & $\begin{array}{c}\mathrm{p} \leq 0.00 \\
1\end{array}$ \\
\hline Median & & & & & & & & & & & & & & & & & \\
\hline Ventilator as & $\operatorname{ated} \mathrm{p}$ & eumo & & & & & & & & & & & & & & & \\
\hline $\begin{array}{c}\text { Unsatisf } \\
\text { actory } \\
\text { knowled } \\
\text { ge }\end{array}$ & 50 & $\begin{array}{c}10 \\
0\end{array}$ & 2 & 4 & 2 & 4 & $\begin{array}{c}x^{2}= \\
87.98\end{array}$ & 50 & $\begin{array}{c}10 \\
0\end{array}$ & 50 & $\begin{array}{c}10 \\
0\end{array}$ & 50 & $\begin{array}{c}10 \\
0\end{array}$ & $\begin{array}{c}\chi^{2}= \\
0.286\end{array}$ & $\begin{array}{c}x^{2}=0.6 \\
44,\end{array}$ & $\chi^{2}=8.64$ & $\begin{array}{c}x^{2}=8.5 \\
7,\end{array}$ \\
\hline $\begin{array}{l}\text { Satisfactory } \\
\text { knowledge }\end{array}$ & -- & -- & 48 & $\begin{array}{l}9 \\
6 \\
\end{array}$ & 48 & $\begin{array}{l}9 \\
6 \\
\end{array}$ & $\mathrm{p} \leq 0.001$ & --- & --- & -- & -- & --- & - & $\mathrm{p} \leq 0.867$ & $\begin{array}{c}\mathrm{P} \leq 0.52 \\
0\end{array}$ & $\mathrm{p} \leq 0.001$ & $\begin{array}{l}\mathrm{p} \leq 0.00 \\
1\end{array}$ \\
\hline Median & & & & & & & & & & & & & & & & & \\
\hline Mechanical v & lator & & & & & & & & & & & & & & & & \\
\hline $\begin{array}{c}\text { Unsatisf } \\
\text { actory } \\
\text { knowled } \\
\text { ge }\end{array}$ & 50 & $\begin{array}{c}10 \\
0\end{array}$ & 5 & $\begin{array}{l}1 \\
0\end{array}$ & 11 & $\begin{array}{l}2 \\
2\end{array}$ & $\chi^{2}=84.31$ & 50 & $\begin{array}{c}10 \\
0\end{array}$ & 50 & $\begin{array}{c}10 \\
0\end{array}$ & 50 & $\begin{array}{l}10 \\
0\end{array}$ & $\chi^{2}=0.200$ & $\begin{array}{c}x^{2}=0.2 \\
50,\end{array}$ & $\chi 2=8.42$ & $\begin{array}{l}x 2=8.1 \\
25,\end{array}$ \\
\hline $\begin{array}{l}\begin{array}{l}\text { Satisfactory } \\
\text { knowledge }\end{array} \\
\end{array}$ & -- & -- & 45 & $\begin{array}{l}9 \\
0 \\
\end{array}$ & 39 & $\begin{array}{l}7 \\
8 \\
\end{array}$ & $\mathrm{p} \leq 0.001$ & --- & - & - & -- & - & - & $\mathrm{p} \leq 0.905$ & $\begin{array}{l}\mathrm{p} \leq 0 \\
882\end{array}$ & $\mathrm{p} \leq 0.001$ & $\begin{array}{l}\mathrm{p} \leq 0.00 \\
1\end{array}$ \\
\hline Median & & 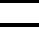 & & & & & & & & & & & & & & & \\
\hline The rendered & rsing & are fo & ritica & ill p & ients & & & & & & & & & & & & \\
\hline $\begin{array}{l}\text { Unsatisf } \\
\text { actory } \\
\text { knowled } \\
\text { ge }\end{array}$ & 50 & $\begin{array}{c}10 \\
0\end{array}$ & 5 & $\begin{array}{l}1 \\
0\end{array}$ & 10 & $\begin{array}{l}2 \\
0\end{array}$ & $\chi^{2}=92.19$ & 49 & 98 & 49 & 98 & 50 & $\begin{array}{c}10 \\
0\end{array}$ & $\chi^{2}=1.355$ & $\begin{array}{c}x^{2}=0.7 \\
65,\end{array}$ & $\chi^{2}=8.28$ & $\begin{array}{c}x_{2}=8.2 \\
7,\end{array}$ \\
\hline $\begin{array}{l}\text { Satisfactory } \\
\text { knowledge }\end{array}$ & -- & -- & 45 & $\begin{array}{l}9 \\
0 \\
\end{array}$ & 40 & $\begin{array}{l}8 \\
0 \\
\end{array}$ & $\mathrm{p} \leq 0.001$ & 1 & 2 & 1 & 2 & - & - & $\mathrm{p} \leq 0.508$ & $\begin{array}{l}\mathrm{p} \leq 0.44 \\
4\end{array}$ & $\mathrm{p} \leq 0.001$ & $\begin{array}{l}\mathrm{p} \leq 0.00 \\
1\end{array}$ \\
\hline Median & & & & & 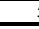 & & & & & & & & & & & & \\
\hline
\end{tabular}

Effectiveness of the educational program on participants' knowledge regarding care bundle on prevention of ventilator-associated pneumonia among intensive care unit nurses:Friedman test was used to examine the difference in knowledge level among the participants in the study and the control groups after 3 months of implementing the educational program. The results showed that there was a highly statistically significant difference in knowledge level regarding all knowledge categories in study group, in contrast no or minimal significant difference in control group. At baseline, the participants in both groups reported similar unsatisfactory knowledge level 
regarding respiratory system, Mechanical ventilator, its functions, VAP, and the rendered nursing care for critically ill patients $(78 \%, \quad 100 \%, 100 \%, \quad 100 \%$, $100 \%$ respectively) as portrayed in Table (2). In contrast at immediate and 3 months post-tests, this unsatisfactory knowledge level improved in the study group to satisfactory one $(84 \%, 78 \%$, $44 \%, \quad 96 \%, \quad 80 \%$ respectively) but remained the same at control group. Furthermore, Friedman test results indicated very highly statistically

Table (3): Comparison between study and control group according to the total knowledge level toward ventilator associated pneumonia (VAP) bundle pre, immediate, 3 months post program (continued.....):

\begin{tabular}{|c|c|c|c|c|c|c|c|c|c|c|c|c|c|c|c|c|c|}
\hline \multirow{4}{*}{ Knowledge level } & \multicolumn{17}{|c|}{ Nurses No=100 } \\
\hline & \multicolumn{6}{|c|}{$\begin{array}{c}\text { Study group } \\
(\mathbf{N}=50)\end{array}$} & \multirow{3}{*}{$\begin{array}{c}\text { Test of } \\
\text { significa } \\
\text { nce } \\
\chi^{2} \\
\text { P }\end{array}$} & \multicolumn{6}{|c|}{$\begin{array}{c}\text { Control group } \\
(\mathrm{N}=50)\end{array}$} & \multirow{3}{*}{$\begin{array}{c}\text { Test of } \\
\text { significa } \\
\text { nce } \\
\chi^{2} \\
P\end{array}$} & \multicolumn{3}{|c|}{ (Study vs Control group) } \\
\hline & \multicolumn{2}{|c|}{ Pre } & \multicolumn{2}{|c|}{$\begin{array}{c}\text { Immediat } \\
\text { e Post }\end{array}$} & \multicolumn{2}{|c|}{$\begin{array}{c}3 \text { months } \\
\text { post }\end{array}$} & & \multicolumn{2}{|c|}{ Pre } & \multicolumn{2}{|c|}{$\begin{array}{c}\text { Immedia } \\
\text { te Post }\end{array}$} & \multicolumn{2}{|c|}{$\begin{array}{l}3 \text { months } \\
\text { post }\end{array}$} & & \multirow{2}{*}{ Pre } & \multirow{2}{*}{$\begin{array}{l}\text { Immedi } \\
\text { ate } \\
\text { post }\end{array}$} & \multirow{2}{*}{$\begin{array}{c}3 \\
\text { mo } \\
\text { nth } \\
\text { s } \\
\text { pos } \\
\text { t }\end{array}$} \\
\hline & No. & $\%$ & $\begin{array}{l}\mathrm{N} \\
\mathrm{o}\end{array}$ & $\%$ & $\begin{array}{l}\mathrm{N} \\
\mathrm{o} .\end{array}$ & $\%$ & & $\begin{array}{l}\mathrm{N} \\
\mathrm{o} .\end{array}$ & $\%$ & $\begin{array}{l}\mathrm{N} \\
\mathrm{o} .\end{array}$ & $\%$ & $\begin{array}{l}\mathrm{N} \\
\mathrm{o}\end{array}$ & $\%$ & & & & \\
\hline \multicolumn{18}{|c|}{ The rendered nursing care for endotracheal tube } \\
\hline $\begin{array}{l}\text { Unsatisfactory } \\
\text { knowledge }\end{array}$ & 49 & $\begin{array}{l}9 \\
8 \\
\end{array}$ & 3 & 6 & 4 & 8 & \multirow{3}{*}{$\begin{array}{c}\chi^{2}=88.03 \\
\mathrm{p} \leq 0.001\end{array}$} & 49 & 98 & 49 & $\begin{array}{l}9 \\
8 \\
\end{array}$ & 48 & $\begin{array}{l}9 \\
6 \\
\end{array}$ & \multirow{3}{*}{$\begin{array}{l}\chi^{2}=4.68, \\
\mathrm{p} \leq 0.096\end{array}$} & \multirow{3}{*}{$\begin{array}{c}\chi^{2}=0.6 \\
25 \\
\mathrm{p} \leq 0.53 \\
2\end{array}$} & \multirow{3}{*}{$\begin{array}{l}\chi 2=8.56 \\
\mathrm{p} \leq 0.001\end{array}$} & $\begin{array}{l}\chi^{2}= \\
8.4\end{array}$ \\
\hline $\begin{array}{c}\text { Satisfactory } \\
\text { nowledge }\end{array}$ & 1 & 2 & 47 & 94 & 46 & 92 & & 1 & 2 & 1 & 2 & 2 & 4 & & & & $\begin{array}{c}8, \\
p \leq 0\end{array}$ \\
\hline Median & \multicolumn{2}{|c|}{11.5} & \multicolumn{2}{|c|}{23} & \multicolumn{2}{|c|}{22} & & \multicolumn{2}{|c|}{10} & \multicolumn{2}{|c|}{10} & \multicolumn{2}{|c|}{11} & & & & 1 \\
\hline \multicolumn{18}{|c|}{ The rendered nursing care during Endotracheal tube (ETT) suctioning } \\
\hline $\begin{array}{c}\text { Unsatisfactory } \\
\text { knowledge }\end{array}$ & 37 & $\begin{array}{l}7 \\
4 \\
\end{array}$ & --- & --- & -- & -- & & 38 & 76 & 37 & $\begin{array}{l}7 \\
4 \\
\end{array}$ & 36 & $\begin{array}{l}7 \\
2 \\
\end{array}$ & & $\chi^{2}=0.9$ & & $\begin{array}{l}\chi^{2}= \\
8.0\end{array}$ \\
\hline $\begin{array}{l}\text { Satisfactory } \\
\text { knowledge }\end{array}$ & 13 & $\begin{array}{l}2 \\
6 \\
\end{array}$ & 50 & $\begin{array}{c}10 \\
0 \\
\end{array}$ & 50 & $\begin{array}{c}10 \\
0 \\
\end{array}$ & $\begin{array}{c}76.09 \\
\mathrm{p} \leq 0.001\end{array}$ & 12 & 24 & 13 & $\begin{array}{l}2 \\
6 \\
\end{array}$ & 14 & $\begin{array}{l}2 \\
8 \\
\end{array}$ & $\begin{array}{l}\chi^{2}=1.09, \\
\mathrm{p} \leq 0.579\end{array}$ & $\begin{array}{c}10, \\
p \leq 0.36\end{array}$ & $\begin{array}{l}\chi^{2}=8.32, \\
\mathrm{p} \leq 0.001\end{array}$ & $\begin{array}{c}3, \\
p \leq 0\end{array}$ \\
\hline Median & 8 & & & & & & & & & & & & & & & & 1 \\
\hline Blood gases analysi & & & & & & & & & & & & & & & & & \\
\hline $\begin{array}{c}\text { Unsatisfactory } \\
\text { knowledge }\end{array}$ & 40 & $\begin{array}{l}8 \\
0 \\
\end{array}$ & 2 & 4 & 3 & 6 & & 42 & 84 & 39 & $\begin{array}{l}7 \\
8 \\
\end{array}$ & 41 & $\begin{array}{l}8 \\
2 \\
\end{array}$ & & $x^{2}=0.6$ & & $\begin{array}{l}x^{2}= \\
7.9\end{array}$ \\
\hline $\begin{array}{l}\text { Satisfactory } \\
\text { knowledge }\end{array}$ & 10 & $\begin{array}{l}2 \\
0 \\
\end{array}$ & 48 & 96 & 47 & 94 & $\mathrm{p} \leq 0.001$ & 8 & 16 & 11 & $\begin{array}{l}2 \\
2 \\
\end{array}$ & 9 & $\begin{array}{l}1 \\
8 \\
\end{array}$ & $\begin{array}{l}\chi^{2}=2.88, \\
\mathrm{p} \leq 0.236\end{array}$ & $\begin{array}{c}73, \\
p \leq 0.50\end{array}$ & $\begin{array}{l}\chi^{2}=8.39 \\
\mathrm{p} \leq 0.001\end{array}$ & $\begin{array}{c}3, \\
p \leq 0\end{array}$ \\
\hline Median & 2 & & & & & & & & & & & & & & & & 1 \\
\hline & & & & & & & & & & & & & & & & asogastric & \\
\hline $\begin{array}{c}\text { Unsatisfactory } \\
\text { knowledge }\end{array}$ & 44 & $\begin{array}{l}8 \\
8 \\
\end{array}$ & 2 & 4 & 3 & 6 & & 46 & 92 & 46 & $\begin{array}{l}9 \\
2 \\
\end{array}$ & 44 & $\begin{array}{l}8 \\
8 \\
\end{array}$ & & $x^{2}=0.0$ & & $\begin{array}{l}x^{2}= \\
7.8\end{array}$ \\
\hline $\begin{array}{l}\text { Satisfactory } \\
\text { knowledge }\end{array}$ & 6 & $\begin{array}{l}1 \\
2 \\
\end{array}$ & 48 & 96 & 47 & 94 & $\mathrm{p} \leq 0.001$ & 4 & 8 & 4 & 8 & 6 & $\begin{array}{l}1 \\
2 \\
\end{array}$ & $\begin{array}{l}\chi^{2}=1.87, \\
p \leq 0.391\end{array}$ & $\begin{array}{c}87, \\
p \leq 0.93\end{array}$ & $\begin{array}{l}\chi^{2}=8.07, \\
\mathrm{p} \leq 0.001\end{array}$ & $\begin{array}{c}5, \\
p \leq 0\end{array}$ \\
\hline Median & 2 & & & & & & & & & & & & & & & & $\begin{array}{l}.00 \\
1\end{array}$ \\
\hline Airway care & & & & & & & & & & & & & & & & & \\
\hline $\begin{array}{c}\text { Unsatisfactorykn } \\
\text { owledge }\end{array}$ & 49 & $\begin{array}{l}9 \\
8 \\
\end{array}$ & 4 & 8 & 4 & 8 & & 50 & $\begin{array}{c}10 \\
0 \\
\end{array}$ & 46 & $\begin{array}{l}9 \\
2 \\
\end{array}$ & 47 & $\begin{array}{l}9 \\
4 \\
\end{array}$ & & $\chi^{2}=0.4$ & & $\begin{array}{l}x^{2}= \\
8.3\end{array}$ \\
\hline $\begin{array}{l}\text { Satisfactory } \\
\text { knowledge }\end{array}$ & 1 & 2 & 46 & 92 & 46 & 92 & $\begin{array}{c}90.00 \\
\mathrm{p} \leq 0.001\end{array}$ & - & $\begin{array}{l}-- \\
-- \\
-\end{array}$ & 4 & 8 & 3 & 6 & $\begin{array}{l}\chi^{2}=1.52, \\
\mathrm{p} \leq 0.467\end{array}$ & $\begin{array}{c}47, \\
p \leq 0.65\end{array}$ & $\begin{array}{l}x^{2}=8.19 \\
\mathrm{p} \leq 0.001\end{array}$ & $\begin{array}{c}8 \\
\mathrm{p} \leq 0\end{array}$ \\
\hline Median & & & & & & & & & & & & & & & & & $\begin{array}{c}.00 \\
1\end{array}$ \\
\hline
\end{tabular}


Impact of Educational Program Regarding etc...

Table(3):demonstratesthedistribut ion of participants' knowledge in both groups regarding nursing care for endotracheal tube, nursing care during endotracheal suctioning, blood gases analysis, nasogastric feeding, and airway care at the three study stages. At the baseline assessment, unsatisfactory knowledge level was reported by both study groups regarding all the above mentioned categories. Compared to immediate post-test, this unsatisfactory level elevated in study group but remained diminished in control group: nursing care for endotracheal tube (study group: $94 \%$, and control group: $2 \%$ ); nursing care during Endotracheal suctioning(study group: $100 \%$, and control group: $26 \%$ ); Blood gases analysis (study group: $96 \%$, and control group: 22\%); Nasogastric feedingAirway care (study group: $96 \%$, and control group: $8 \%$ ). Furthermore, Friedman test results indicated very highly statistically differences in the study group regarding all previous mentioned categories (p $\leq \mathbf{0 . 0 0 1 )}$, comparing with the control group results insignificant differences was detected at the three study stages $(\mathbf{P}=0.096, \mathbf{P}=0.579, \mathbf{P}=0.236, \mathbf{P}=0.391$, $\mathbf{P}=0.467$ respectively).

Table(4):Comparison between study and control group according to the total knowledge level toward ventilator associated pneumonia (VAP) bundle pre, immediate, 3 months post program (continued.....):

\begin{tabular}{|c|c|c|c|c|c|c|c|c|c|c|c|c|c|c|c|c|c|}
\hline \multirow{4}{*}{$\begin{array}{l}\text { Knowledge } \\
\text { level }\end{array}$} & \multicolumn{17}{|c|}{ Nurses No=100 } \\
\hline & \multicolumn{6}{|c|}{$\begin{array}{c}\text { Study group } \\
(\mathrm{N}=50)\end{array}$} & \multirow{3}{*}{$\begin{array}{c}\text { Test } \\
\text { of } \\
\text { signif } \\
\text { icanc } \\
\text { e } \\
\chi^{2} \\
\text { P }\end{array}$} & \multicolumn{6}{|c|}{$\begin{array}{c}\begin{array}{c}\text { Control group } \\
(\mathrm{N}=50)\end{array} \\
\end{array}$} & \multirow{3}{*}{$\begin{array}{c}\text { Test of } \\
\text { significan } \\
\text { ce } \\
\chi^{2} \\
\mathbf{P}\end{array}$} & \multicolumn{3}{|c|}{ (Study vs Control group) } \\
\hline & \multicolumn{2}{|c|}{ Pre } & \multicolumn{2}{|c|}{$\begin{array}{c}\text { Immediate } \\
\text { Post }\end{array}$} & \multicolumn{2}{|c|}{$\begin{array}{l}3 \text { months } \\
\text { Post }\end{array}$} & & \multicolumn{2}{|c|}{ Pre } & \multicolumn{2}{|c|}{$\begin{array}{c}\text { Immediat } \\
\text { e Post }\end{array}$} & \multicolumn{2}{|c|}{$\begin{array}{l}3 \text { months } \\
\text { post }\end{array}$} & & \multirow{2}{*}{ Pre } & \multirow{2}{*}{$\begin{array}{c}\text { Immedia } \\
\text { te post }\end{array}$} & \multirow{2}{*}{$\begin{array}{c}3 \\
\text { month } \\
\text { s post }\end{array}$} \\
\hline & No & $\%$ & No & $\%$ & No & $\%$ & & No. & $\%$ & No & $\%$ & No & $\%$ & & & & \\
\hline \multicolumn{18}{|c|}{ The patient mobilization } \\
\hline $\begin{array}{l}\text { Unsatisfact } \\
\text { ory } \\
\text { knowledge }\end{array}$ & 42 & $\begin{array}{l}8 \\
4 \\
\end{array}$ & 1 & 2 & 1 & 2 & \multirow{3}{*}{$\begin{array}{l}x^{2}=84.00, \\
\mathrm{p} \leq 0.001\end{array}$} & 44 & $\begin{array}{l}8 \\
8\end{array}$ & 38 & $\begin{array}{l}7 \\
6 \\
\end{array}$ & 44 & $\begin{array}{l}8 \\
8\end{array}$ & \multirow{3}{*}{$\begin{array}{l}\chi^{2}=5.54, \\
\mathrm{p} \leq 0.06\end{array}$} & \multirow{3}{*}{$\begin{array}{c}\chi^{2}=0.23 \\
4, \\
\mathrm{p} \leq 0.815\end{array}$} & \multirow{3}{*}{$\begin{array}{l}\chi^{2}=7.39 \\
\mathrm{p} \leq 0.001\end{array}$} & \multirow{3}{*}{$\begin{array}{c}x^{2}=8.2 \\
9, \\
\mathrm{p} \leq 0.00 \\
1\end{array}$} \\
\hline $\begin{array}{l}\text { Satisfactory } \\
\text { knowledge }\end{array}$ & 8 & $\begin{array}{l}1 \\
6 \\
\end{array}$ & 49 & 98 & 49 & 98 & & 6 & $\begin{array}{l}1 \\
2 \\
\end{array}$ & 12 & $\begin{array}{l}2 \\
4\end{array}$ & 6 & $\begin{array}{l}1 \\
2\end{array}$ & & & & \\
\hline Median & \multicolumn{2}{|c|}{2} & \multicolumn{2}{|c|}{3} & \multicolumn{2}{|c|}{3} & & \multicolumn{2}{|c|}{2} & \multicolumn{2}{|c|}{2} & \multicolumn{2}{|c|}{2} & & & & \\
\hline \multicolumn{18}{|c|}{ The chest physiotherapy } \\
\hline $\begin{array}{l}\text { Unsatisfact } \\
\text { ory } \\
\text { knowledge }\end{array}$ & 45 & $\begin{array}{l}9 \\
0\end{array}$ & 2 & 4 & 2 & 4 & \multirow{3}{*}{$\begin{array}{r}\chi^{2}=76.47, \\
p \leq 0.001\end{array}$} & 45 & $\begin{array}{l}9 \\
0\end{array}$ & 44 & $\begin{array}{l}8 \\
8\end{array}$ & 41 & $\begin{array}{l}8 \\
2\end{array}$ & & $\chi^{2}=0.22$ & & $x^{2}=7.8$ \\
\hline $\begin{array}{l}\text { Satisfactory } \\
\text { knowledge }\end{array}$ & 5 & $\begin{array}{l}1 \\
0\end{array}$ & 48 & 96 & 48 & 96 & & 5 & $\begin{array}{l}1 \\
0\end{array}$ & 6 & $\begin{array}{l}1 \\
2\end{array}$ & 9 & $\begin{array}{l}1 \\
8\end{array}$ & $\mathrm{p} \leq 0.339$ & $\begin{array}{c}0, \\
\mathrm{p} \leq 0.826\end{array}$ & $\mathrm{p} \leq 0.001$ & $\begin{array}{c}\mathrm{p} \leq 0.00 \\
1\end{array}$ \\
\hline Median & & & & & & & & & & 3 & & 5 & & & & & \\
\hline The mechan & Ivent & tor ci & uits s & ilizat & & & & & & & & & & & & & \\
\hline $\begin{array}{l}\text { Unsatisfact } \\
\text { ory } \\
\text { knowledge }\end{array}$ & 40 & $\begin{array}{l}8 \\
0\end{array}$ & --- & --- & --- & -- & & 42 & $\begin{array}{l}8 \\
4\end{array}$ & 39 & $\begin{array}{l}7 \\
8\end{array}$ & 41 & $\begin{array}{l}8 \\
2\end{array}$ & & & & \\
\hline $\begin{array}{l}\text { Satisfactory } \\
\text { knowledge }\end{array}$ & 10 & $\begin{array}{l}2 \\
0 \\
\end{array}$ & 50 & $\begin{array}{c}10 \\
0\end{array}$ & 50 & $\begin{array}{c}10 \\
0\end{array}$ & $\begin{aligned} \chi^{2}=73.57, \\
\mathrm{p} \leq 0.001\end{aligned}$ & 8 & $\begin{array}{l}1 \\
6\end{array}$ & 11 & $\begin{array}{l}2 \\
2\end{array}$ & 9 & $\begin{array}{l}1 \\
8\end{array}$ & $\begin{array}{l}\chi^{2}=1.83, \\
p \leq 0.399\end{array}$ & $\begin{array}{l}5 \\
\mathrm{p} \leq 0.671\end{array}$ & $\begin{array}{l}x^{2}=7.72, \\
\mathrm{p} \leq 0.001\end{array}$ & $\begin{array}{c}9, \\
\mathrm{p} \leq 0.00 \\
1\end{array}$ \\
\hline Median & & & & & & & & & & & & 3. & & & & & \\
\hline Total knowl & e leve & & & & & & & & & & & & & & & & \\
\hline $\begin{array}{l}\text { Unsatisfact } \\
\text { ory } \\
\text { knowledge }\end{array}$ & 49 & $\begin{array}{l}9 \\
8\end{array}$ & 2 & 4 & 2 & 4 & & 49 & $\begin{array}{l}9 \\
8\end{array}$ & 49 & $\begin{array}{l}9 \\
8\end{array}$ & 48 & $\begin{array}{l}9 \\
6\end{array}$ & & & & $x^{2}=8.6$ \\
\hline $\begin{array}{l}\begin{array}{l}\text { Satisfactory } \\
\text { knowledge }\end{array} \\
\end{array}$ & 1 & 2 & 48 & 96 & 48 & 96 & $\begin{array}{c}\chi=93.48, \\
\mathrm{p} \leq 0.001\end{array}$ & 1 & 2 & 1 & 2 & 2 & 4 & $\begin{array}{c}\chi=4.81, \\
\mathrm{p} \leq 0.09\end{array}$ & $\begin{array}{c}0, \\
\mathrm{p} \leq 0.562\end{array}$ & $\begin{array}{l}x_{2}^{2}=8.62, \\
\mathrm{p} \leq 0.001\end{array}$ & $\begin{array}{c}0, \\
\mathrm{p} \leq 0.00 \\
1\end{array}$ \\
\hline Median & 4 & & & & & & & & & 46 & & 4 & & & & & \\
\hline
\end{tabular}

Table (4): portraysthe distribution of participants' knowledge in both groups regarding nursing care for patient mobilization, chest physiotherapy, and mechanical ventilator circuits sterilization at the three study stages. At the initial survey, unsatisfactory knowledge level was detected by both 
study groups regarding all the previous mentioned categories. Compared to 3 months post-test, the study group showed satisfactory level, in contrast to, the control group who reported the lower satisfactory level: patient mobilization(study group: $98 \%$, and control group: $12 \%$ ); chest physiotherapy (study group: 96\%, and control group: $18 \%)$; mechanical ventilator circuits sterilization(study group: 100\%, and control group: 18\%). Furthermore, Friedman test results indicated very highly statistically improvements in the study group regarding all the previously mentioned categories $(\mathrm{p} \leq 0.001)$, comparing with the control group results insignificant differences was indicated at the three study stages $(\mathrm{P}=0.06, \mathrm{P}=0.339$, $\mathrm{P}=0.399$, respectively).

Figure (1):Comparison between study and control group according to the total knowledge level toward ventilator associated pneumonia (VAP) bundle pre, immediate, 3 months post program

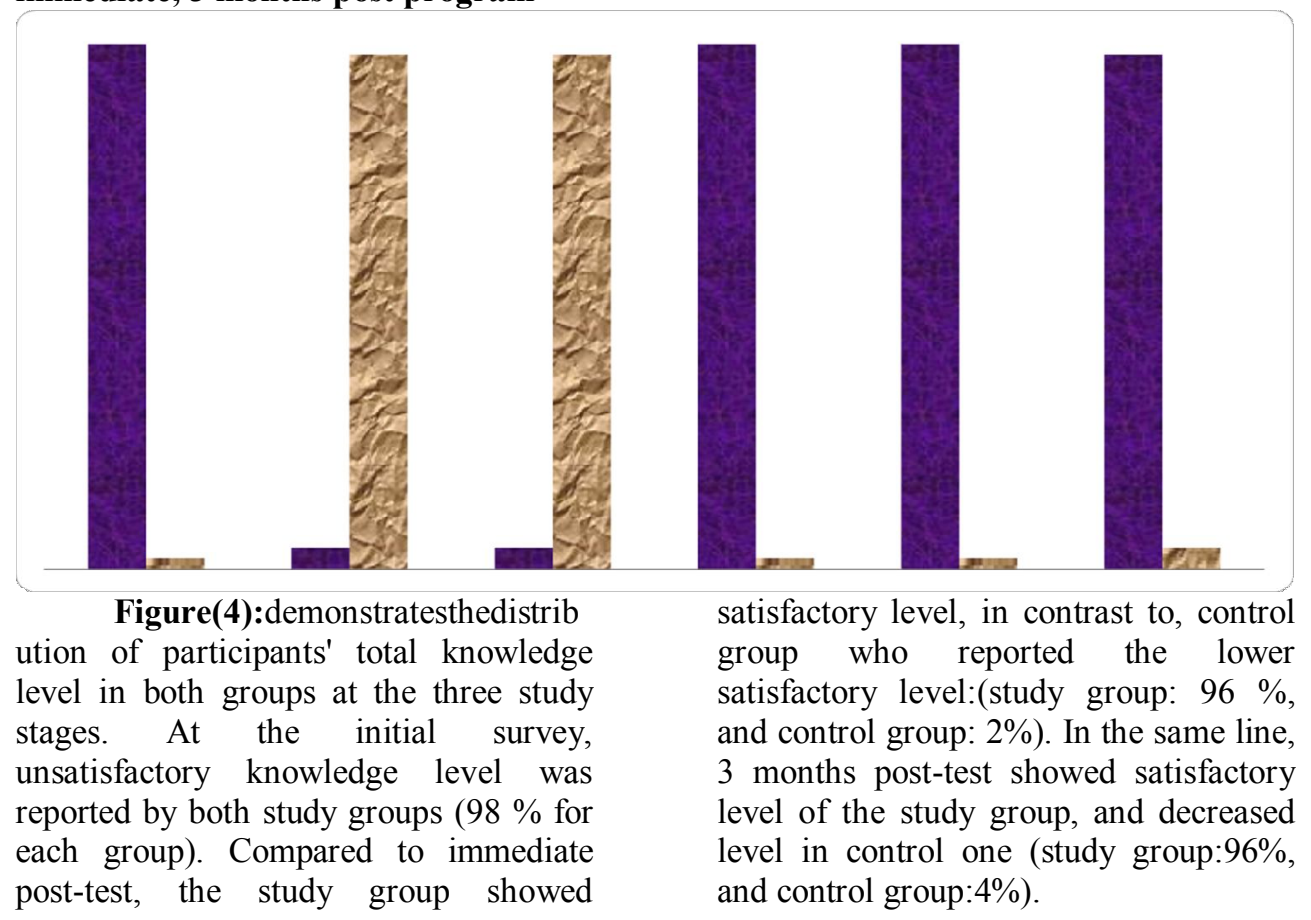

results indicated very highly statistical improvements in study group median score from baseline (48) to immediate post-test (93) and 3 months post-test (91)at $\left(\chi^{\mathbf{2}}=\mathbf{9 3 . 4 8}, \mathbf{P} \leq \mathbf{0 . 0 0 1}\right)$, in contrast to the control group results showed insignificant differences at $\left(\chi^{2}=\mathbf{4 . 8 1}\right.$, $\mathbf{P}=\mathbf{0 . 0 9}$ ), with minimal change of median score from baseline (46.5) to immediate post-test (46.5) and 3 months post-test (48)at the three study stages, in addition, to chi square test demonstrated statistical differences between immediate post and 3 months post-test percentages at ( $\mathbf{P}$ $\leq \mathbf{0 . 0 0 1 )}$. 
Impact of Educational Program Regarding etc...

Table (5): Association of the Socio-demographic and occupational characteristics of the studygroup and their total knowledge level about ventilator associated pneumonia (VAP) bundle pre, immediate, 3 months post program:

\begin{tabular}{|c|c|c|c|c|c|c|c|c|c|c|c|c|c|c|c|c|}
\hline \multirow{4}{*}{\multicolumn{2}{|c|}{$\begin{array}{c}\text { Nurses' socio- } \\
\text { demographic and } \\
\text { occupational variables }\end{array}$}} & \multicolumn{12}{|c|}{ Total knowledge level $N o=50$} & \multirow{2}{*}{\multicolumn{3}{|c|}{$\begin{array}{c}\text { Test of Significance } \\
\begin{array}{c}\text { Chi square, Monte Carlo\& or } \\
\text { fisher exact test }\end{array}\end{array}$}} \\
\hline & & \multicolumn{4}{|c|}{ Pre } & \multicolumn{4}{|c|}{ Immediate post } & \multicolumn{4}{|c|}{3 months post } & & & \\
\hline & & \multicolumn{2}{|c|}{$\begin{array}{l}\text { Unsatisfactory } \\
\text { knowledge } \\
\mathrm{N}=49\end{array}$} & \multicolumn{2}{|c|}{$\begin{array}{c}\begin{array}{c}\text { Satisfactory } \\
\text { knowledge } \\
\mathrm{N}=1\end{array} \\
\end{array}$} & \multicolumn{2}{|c|}{$\begin{array}{c}\text { Unsatisfactory } \\
\text { knowledge } \\
\mathrm{N}=2\end{array}$} & \multicolumn{2}{|c|}{$\begin{array}{c}\begin{array}{c}\text { Satisfactory } \\
\text { knowledge } \\
\mathrm{N}=48\end{array} \\
\end{array}$} & \multicolumn{2}{|c|}{$\begin{array}{c}\begin{array}{c}\text { Unsatisfactor } \\
\text { y knowledge } \\
\mathrm{N}=2\end{array} \\
\end{array}$} & \multicolumn{2}{|c|}{$\begin{array}{c}\begin{array}{l}\text { Satisfactory } \\
\text { knowledge } \\
\mathrm{N}=48\end{array} \\
\end{array}$} & \multirow{2}{*}{$\frac{\text { Pre }}{{ }^{2} \& P_{1}}$} & \multirow{2}{*}{$\frac{\begin{array}{c}\text { Immediat } \\
\mathrm{e}\end{array}}{{ }_{2}^{2} \& \mathrm{P}_{2}}$} & \multirow{2}{*}{$\begin{array}{c}\begin{array}{c}3 \\
\text { month } \\
\text { s post }\end{array} \\
{ }_{3}^{2} \& \mathrm{P}_{3}\end{array}$} \\
\hline & & No. & $\%$ & No. & $\%$ & No. & $\%$ & No. & $\%$ & No & $\%$ & No. & $\%$ & & & \\
\hline \multirow[t]{3}{*}{ Nurses' age } & $\begin{array}{l}20- \\
<30 \mathrm{ys}\end{array}$ & 16 & 62 & -.-. & -- & 1 & 2 & 15 & 30 & 1 & 2 & 15 & 30 & \multirow{3}{*}{$\begin{array}{l}\mathrm{MC} \\
\& \\
\therefore 119\end{array}$} & \multirow{3}{*}{$\begin{array}{c}\mathrm{MC} \\
\& \\
1 .\end{array}$} & \multirow{3}{*}{\begin{tabular}{c}
$\mathrm{MC}$ \\
$\&$ \\
$\therefore$ \\
\hdashline
\end{tabular}} \\
\hline & $\begin{array}{l}30- \\
<40 \mathrm{ys}\end{array}$ & 24 & 48 & --- & - & 1 & 2 & 23 & 46 & 1 & 2 & 23 & 46 & & & \\
\hline & $\begin{array}{c}40- \\
<50 y s\end{array}$ & 9 & 18 & 1 & 2 & --- & $\cdots$ & 10 & 20 & $\cdots$ & $-\cdots$ & 10 & 20 & & & \\
\hline \multirow{3}{*}{$\begin{array}{l}\text { Nurses' } \\
\text { qualificatio } \\
\mathrm{n}\end{array}$} & $\begin{array}{c}\text { Diploma } \\
\text { of } \\
\text { nursing }\end{array}$ & 7 & 14 & 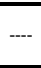 & $\because$ & 1 & 2 & 6 & 12 & 1 & 2 & 6 & 12 & \multirow[t]{3}{*}{$\begin{array}{c}\mathrm{MC} \\
\& 0.45 \\
4\end{array}$} & \multirow[t]{3}{*}{$\begin{array}{c}\mathrm{MC} \\
\& \\
0.208\end{array}$} & \multirow[t]{3}{*}{$\begin{array}{c}\mathrm{MC} \\
\& \\
0.208\end{array}$} \\
\hline & $\begin{array}{c}\begin{array}{c}\text { Nursing } \\
\text { diploma } \\
\text { and } \\
\text { specialist }\end{array} \\
\end{array}$ & 27 & 54 & $\cdots$ & - & -- & -- & 27 & 54 & -- & -- & 27 & 54 & & & \\
\hline & $\begin{array}{l}\text { Technica } \\
\text { 1 Nursing } \\
\text { Institute }\end{array}$ & 15 & 30 & 1 & 2 & 1 & 2 & 15 & 30 & 1 & 2 & 15 & 30 & & & \\
\hline \multirow[t]{2}{*}{$\begin{array}{c}\text { Experience } \\
\text { years }\end{array}$} & $5-<10 \mathrm{ys}$ & 42 & 84 & -- & --- & 2 & 4 & 40 & 80 & 2 & 4 & 40 & 80 & \multirow{2}{*}{$\begin{array}{c}\mathrm{FE} \\
\& 0.16 \\
0\end{array}$} & \multirow[t]{2}{*}{$\begin{array}{c}\mathrm{FE} \\
\& 1.00\end{array}$} & \multirow[t]{2}{*}{$\begin{array}{c}\mathrm{FE} \\
\& 1.00\end{array}$} \\
\hline & $\geq 10 \mathrm{ys}$ & 7 & 14 & 1 & 2 & -- & -- & 8 & 16 & -- & -- & 8 & 16 & & & \\
\hline \multirow{2}{*}{$\begin{array}{c}\text { Previous } \\
\text { attendance } \\
\text { of training } \\
\text { program }\end{array}$} & NO & 45 & 90 & -- & --- & 2 & 4 & 43 & 86 & 2 & 4 & 43 & 86 & \multirow{2}{*}{$\begin{array}{c}\mathrm{MC} \\
\& 0.10 \\
0\end{array}$} & \multirow{2}{*}{$\begin{array}{l}\mathrm{FE} \\
1 \cdots \&\end{array}$} & \multirow{2}{*}{$\begin{array}{c}\mathrm{FE} \\
\because \cdots \&\end{array}$} \\
\hline & Yes & 4 & 8 & 1 & 2 & --- & --- & 5 & 10 & -.- & -.- & 5 & 10 & & & \\
\hline
\end{tabular}

(*) Statistically significant at $\mathrm{p} \leq 0.05, \chi^{2}$ $=$ chi square, MC: Monte Carlo test, FE: Fisher Exact test

Table (5): shows the association of the socio-demographic and occupational characteristics of the study group and their total knowledge level at the three study phases. Regarding the nurses' age, the Monte Carlo results indicated insignificant relationship between nurses' age and their total knowledge level at the three study phases $(\mathrm{P}=0.189, \mathrm{P}=1.00, \mathrm{P}=1.00 \quad$ respectively).
In accordance with nurses' qualification, the Monte Carlo results showed no significant relationship between nurses' qualification and their total knowledge level at the three study stages ( $\mathrm{P}=0.454, \mathrm{P}=0.208, \mathrm{P}=0.208$ respectively). Concerning experience years, fisher exact test indicated insignificant differences throughout the study phases at $\quad(\mathrm{P}=0.320, \quad \mathrm{P}=0.320, \quad \mathrm{P}=0.542$ respectively). For previous attendance of training program, the exact tests showed similar insignificant results at $(\mathrm{P}=1.00)$. 
Table (6): Association of the Socio-demographic and occupational characteristics of thecontrolgroup and their total knowledge level about ventilator associated pneumonia (VAP) bundle pre, immediate, 3 months post program:

\begin{tabular}{|c|c|c|c|c|c|c|c|c|c|c|c|c|c|c|c|c|}
\hline \multirow{4}{*}{\multicolumn{2}{|c|}{$\begin{array}{l}\text { Nurses' socio- } \\
\text { demographic and } \\
\text { occupational } \\
\text { variables }\end{array}$}} & \multicolumn{12}{|c|}{ Total knowledge level $N o=50$} & \multirow{2}{*}{\multicolumn{3}{|c|}{$\begin{array}{c}\text { Test of Significance } \\
\text { Chi square, Monte Carlo\& } \\
\text { or fisher exact test }\end{array}$}} \\
\hline & & \multicolumn{4}{|c|}{ Pre } & \multicolumn{4}{|c|}{ Immediate post } & \multicolumn{4}{|c|}{3 months post } & & & \\
\hline & & \multicolumn{2}{|c|}{$\begin{array}{c}\text { Unsatisfact } \\
\text { ory } \\
\text { knowledge } \\
\mathrm{N}=49\end{array}$} & \multicolumn{2}{|c|}{$\begin{array}{c}\text { Satisfacto } \\
\text { ry } \\
\text { knowledg } \\
\mathrm{e} \\
\mathrm{N}=1\end{array}$} & \multicolumn{2}{|c|}{$\begin{array}{c}\text { Unsatisfact } \\
\text { ory } \\
\text { knowledge } \\
\mathrm{N}=49\end{array}$} & \multicolumn{2}{|c|}{$\begin{array}{c}\text { Satisfacto } \\
\text { ry } \\
\text { knowledg } \\
\mathrm{e} \\
\mathrm{N}=1\end{array}$} & \multicolumn{2}{|c|}{$\begin{array}{c}\text { Unsatisfact } \\
\text { ory } \\
\text { knowledge } \\
\mathrm{N}=48\end{array}$} & \multicolumn{2}{|c|}{$\begin{array}{l}\text { Satisfactory } \\
\text { knowledge } \\
\mathrm{N}=2\end{array}$} & \multirow{2}{*}{$\begin{array}{l}\text { Pre } \\
\\
{ }_{1}^{2} \& \\
\mathbf{P}_{1}\end{array}$} & \multirow{2}{*}{$\begin{array}{c}\substack{\text { Immedi } \\
\text { ate }} \\
{ }_{2}^{2} \& P_{2}\end{array}$} & \multirow{2}{*}{$\begin{array}{c}\begin{array}{c}3 \\
\text { mont } \\
\text { hs } \\
\text { post }\end{array} \\
{ }_{3}^{2} \& \\
\mathbf{P}_{3}\end{array}$} \\
\hline & & No. & $\%$ & No & $\%$ & No. & $\%$ & $\overline{\text { No }}$ & $\%$ & No. & $\%$ & No. & $\%$ & & & \\
\hline \multirow{3}{*}{$\begin{array}{l}\text { Nur } \\
\text { ses' } \\
\text { age }\end{array}$} & $20-<30 \mathrm{ys}$ & 15 & 30 & -- & $\begin{array}{l}-- \\
--\end{array}$ & 15 & 30 & -- & $\begin{array}{l}-- \\
--\end{array}$ & 15 & 30 & ---- & -- & \multirow{3}{*}{$\begin{array}{l}\mathrm{MC} \\
\& \\
r T \\
r\end{array}$} & \multirow{3}{*}{$\begin{array}{c}\mathrm{MC} \\
\cdot . r 9 \% \&\end{array}$} & \multirow{3}{*}{$\begin{array}{l}\mathrm{MC} \\
\& \\
\ddots V T \\
r\end{array}$} \\
\hline & $30-<40 \mathrm{ys}$ & 22 & 44 & -- & -- & 22 & 44 & - & -- & 21 & 42 & 1 & 2 & & & \\
\hline & $40-<50$ ys & 12 & 24 & 1 & 2 & 12 & 24 & 1 & 2 & 12 & 24 & 1 & 2 & & & \\
\hline \multirow{3}{*}{$\begin{array}{l}\text { Nurses } \\
\text { qualifi } \\
\text { cation }\end{array}$} & $\begin{array}{l}\text { Diploma of } \\
\text { nursing }\end{array}$ & 12 & 24 & $-\overline{-}$ & -- & 12 & 24 & -- & -- & 12 & 24 & --- & -- & \multirow{3}{*}{$\begin{array}{c}\mathrm{MC} \\
\& \\
0.594\end{array}$} & \multirow{3}{*}{$\begin{array}{c}\mathrm{MC} \\
\& \\
0.594\end{array}$} & \multirow{3}{*}{$\begin{array}{c}\mathrm{MC} \\
\& \\
1.00\end{array}$} \\
\hline & $\begin{array}{l}\text { Nursing } \\
\text { diploma } \\
\text { and } \\
\text { specialist }\end{array}$ & 20 & 40 & -- & -- & 20 & 40 & - & -- & 19 & 38 & 1 & 2 & & & \\
\hline & $\begin{array}{l}\text { Technical } \\
\text { Nursing } \\
\text { Institute }\end{array}$ & 17 & 34 & 1 & 2 & 17 & 34 & 1 & 2 & 17 & 34 & 1 & 2 & & & \\
\hline \multirow{2}{*}{$\begin{array}{l}\begin{array}{l}\text { Experi } \\
\text { ence } \\
\text { years }\end{array} \\
\end{array}$} & $5-<10 \mathrm{ys}$ & 34 & 68 & --- & $\begin{array}{l}-- \\
--\end{array}$ & 34 & 68 & $\begin{array}{l}--- \\
-\end{array}$ & $\begin{array}{l}-- \\
--\end{array}$ & 33 & 66 & 1 & 2 & \multirow{2}{*}{$\begin{array}{c}\mathrm{FE} \\
\& 0.3 \\
20\end{array}$} & \multirow[t]{2}{*}{$\begin{array}{c}\mathrm{FE} \\
\& 0.320\end{array}$} & \multirow{2}{*}{$\begin{array}{c}\mathrm{FE} \\
\& 0.5 \\
42\end{array}$} \\
\hline & $\geq 10 \mathrm{ys}$ & 15 & 30 & 1 & 2 & 15 & 30 & 1 & 2 & 15 & 30 & 1 & 2 & & & \\
\hline \multirow{2}{*}{$\begin{array}{l}\text { Previo } \\
\text { attend: } \\
\text { e } \\
\text { trainin } \\
\text { progra }\end{array}$} & NO & 39 & 78 & - & -- & 39 & 78 & - & -- & 38 & 76 & 1 & 2 & \multirow{2}{*}{$\begin{array}{c}\mathrm{FE} \\
\& \\
0.220\end{array}$} & \multirow{2}{*}{$\begin{array}{c}\mathrm{FE} \\
\& \\
0.220\end{array}$} & \multirow{2}{*}{$\begin{array}{l}\mathrm{FE} \\
\& \\
\because{ }_{0}\end{array}$} \\
\hline & Yes & 10 & 20 & 1 & 2 & 10 & 20 & 1 & 2 & 10 & 20 & 1 & 2 & & & \\
\hline
\end{tabular}

(*) Statistically significant at $p \leq 0.05, \chi^{2}$

= chi square, MC: Monte Carlo test, FE:

Fisher Exact test

Table (6): shows the association of the socio-demographic and occupational characteristics of the control group and their total knowledge level at the three study phases. Regarding the nurses' age, the Monte Carlo results demonstrated insignificant relationship between age and total knowledge level at the three study phases $(\mathrm{P}=0.263$, $\mathrm{P}=0.265, \quad \mathrm{P}=0.733$ respectively). As regards to nurses' qualification, the Monte Carlo results showed no significant relationship between qualifications and total knowledge level at the three study stages $(\mathrm{P}=0.594, \mathrm{P}=0.594, \mathrm{P}=1.00$ respectively). In relation to experience years, fisher exact test indicated insignificant differences throughout the study phases at $\quad(\mathrm{P}=0.320, \quad \mathrm{P}=0.320, \quad \mathrm{P}=0.542$ respectively). For previous attendance of training program, the fisher exact tests showed similar insignificant results at $(\mathrm{P}=0.220, \quad \mathrm{P}=0.220, \quad \mathrm{P}=0.395$ respectively).

\section{Discussion:}

which is related to severe morbidity and high care costs. VAP's pathophysiology, epidemiology, diagnosis, and prevention have been researched widely for decades, but no specific preventive strategy has yet emerged. This study was conducted to examine the effect of education given to nurses to reduce the VAP rate in adult ICUs.

Regarding the demographic and occupational characteristics, chi square test results indicated no statistically significant difference in demographic characteristics between the study and control groups, which indicates the homogeneity of the both groups. This 
homogeneity is the basic requirements for any case-control study as revealed by consistent study of Oner Cengiz, \& Kanan, (2019), who reported that "there was no statistically significant difference in descriptive characteristics between the study and control groups".

According to Akin Korhan, Hakverdioglu Yont, Parlar Kilic, \& Uzelli (2014), increasing ICU nurses' knowledge of VAP and care bundle activities are the effective way to prevent VAP in the care environment. In the literature, where ICU nurses' knowledge of VAP prevention was evaluated, nurses' knowledge of VAP was reported to be insufficient (Maurya, Mishra, Azim, Baronia, \& Gurjar, 2016; AlSayaghi, 2014; Jansson, Ala-Kokko, Ylipalosaari, Syrjälä ,\& Kyngäs, 2013). In the current study, the total median scores before the first education in the study group and the median scores at the first measurement in the control group were detected to be low on the knowledge test. This result comes in agreements with another study of Dipanjali, Shivananda, \& Yashoda, (2021) entitled as "the effectiveness of the educational intervention on the knowledge and practice of the staff nurses on prevention of VAP in neonates", which showed lower scores of both study groups at pre-test, and showed improvement in the knowledge scores of the nurses after the educational sessions $(p \leq 0.001)$. Moreover, in the study of Meherali, Parpio, Ali, \& Javed, (2011) that examine "the effectiveness of a teaching module on nurses' knowledge to practice evidence based guidelines for the prevention of VAP", the post-tests scores were higher than the pre-test scores although there was a slight decrease in the follow up-test scores conducted after four weeks of the intervention as compared to post-test conducted immediately after the intervention (pre-test $=7.8 \pm 2.9$, post-test $1=10.8 \pm 2.0$ \& post-test $2=9.8 \pm 2.1$ ).

Almost 20 years ago, it was well recognized that there is a deficiency in the mechanical ventilation knowledge among the internal medical staff in the United States (Al-Otaibi, 2019). This conclusion is congruent with the current study results related to knowledge of concept of mechanical ventilator, its functions, and the way of its circuits sterilization, as it showed unsatisfactory levels of both study groups at initial assessment which improved later in the study group at immediate and 3 months post-test results after educational intervention. Additionally, these finding compatibles with one large European study that included 318 ICUs, among 11 domains of intensive carerelated individuals, the performance of nurses was lowest in the ventilation domain. The nurses scored an average of $66 \%$ across all 11 domains (Fulbrook, Albarran, Baktoft, \& Sidebottom, 2012)

As regard to the chest physiotherapy and patient mobilization knowledge categories, the current study indicated unsatisfactory level at preassessment phase in both study groups which improved later in study group but not in control group at immediate post and follow up phases. This finding is consistent with Bahgat (2018)and Ramya \& Neethu (2013) who concluded that "more than three quarter of their participants had poor level of total knowledge; pre- intervention which improved to a good level at immediate post and one-month post intervention".

Concerning airway care, ETT suctioning, and rendered NC during suctioning categories, the present study 
revealed that unsatisfactory level at preassessment phase in both study groups which improved later in study group but not in control group at immediate post and follow up tests. This finding compatibles with the study of Abdelazeem, Fashafsheh, \& Fadllalah, (2019), who reported that "The knowledge of the participant regarding ETT and air way care was intermediate pre intervention by $38 \%$ then the result improved to becomes good in post intervention I by $46 \%$ and get increase by $54 \%$ in post intervention II". These finding indicates the hazard of being as a bedside nurse for mechanically ventilated patients with short knowledge about the tools which connecting the patient to mechanical ventilator and airway management. The improvement in these finding from pre to two posttests reflects the good effect of the educational program:

In relation to nasogastric feeding category, present study showed that unsatisfactory level at initial assessment phase in both study groups which elevated later in study group, while not elevated in control group at immediate post and follow up tests. This finding is in the same line with Bedier, Abo ELAta, \& Shehab (2020), who cited that "the study results showed statistically significant improvement in nurses total score regarding nasogastric tube feeding and this improvement was tended to decline but sustainable immediately, post 3 month and 6 month after the program implementation compared to pre-test".

In accordance with the association of socio-demographic,occupational characteristics and the total knowledge level of both nurses groups about VAP. The present study presented insignificant association of total knowledge level with selected demographic and occupational variables. In the same line with the study conducted by Ghimire and Neupane (2018) who presented no statistically significant association between respondents' level of knowledge regarding the prevention of VAP with working experience $(p=0.493)$ and qualification $(p=0.459)$. Similar findings were reported by Rakhi, and Navita, (2020), who demonstrated statistically insignificant relationship between participants' level of knowledge regarding the prevention of VAP with age $(p=0.17)$ and qualification $(p=5.44)$, and work experience $(\mathrm{P}=0.09)$. On the other hand, this finding contradicts with the study of Baby (2012) who revealed that "there was a significant association between the knowledge level with demographic variables like age, religion and marital status at the level of $\mathrm{p}<$ $0.05 "$.

Conclusion: the study concluded that unsatisfactory knowledge was reported by both study groups before education implementation. Compared to immediate post-test, significant improvements in the study group knowledge were detected, in contrast to, control group who didn't show any significant differences. In the same line, 3 months post-test showed satisfactory level of the study group with highly statistically significant differences, and insignificant lower level in the control group.

Recommendations: Based on the results of the present study, the following recommended: Continuous nursing education programs at ICU should be organized within Meniet ELNasr and Dekirnis Central Hospitals. Nurses should add to their daily routine responsibilities the regular reading to update their knowledge. They should always be encouraged to attend scientific workshops and conferences to keep pace 
Impact of Educational Program Regarding etc...

with the rapid growing plenty of knowledge and practice necessary for proper care bundles implementation.

\section{References:}

Mishra, R., \& Rani, N. (2020). Effectiveness of Structured Teaching Program on Knowledge and Practice Regarding Care Bundle on Prevention of Ventilator-Associated Pneumonia among Nurses. Int Arch Nurs Health Care 6:149. doi.org/10.23937/24695823/1510149.

Chithra, R.A., \& Raju, J. (2017). Effect of structured teaching programme on knowledge regarding prevention of ventilator associated pneumonia among critical care nurses. Int $\mathrm{J}$ Health Sci Res, 7(7), 156-163.

Timsit, J.F., Esaied, W., Neuville, M., Bouadma, L.,\& Mourvllier, B. (2017). Update on ventilatorassociated pneumonia. F1000Res 6: 2061.

Bouadma, L., Sonneville, R., Garrouste, O.M., Darmon, M., Souweine, B., et al. (2015). Ventilator-associated events: Prevalence, outcome, and relationship with ventilatorassociated pneumonia. Crit Care Med, 43, 1798:1806.

Institute for Healthcare Improvement. (2020). Bundle up for safety. Retrieved 4 April 2021 from:http://www.ihi.org/Topics/B undles/Pages/default.aspx.

Lavallée, J. F., Gray, T. A., Dumville, J., Russell, W., \& Cullum, N. (2017). The effects of care bundles on patient outcomes: a systematic review and metaanalysis. Implementation science : IS, 12(1), 142. Retrieved 4 April
2021 from:

https://doi.org/10.1186/s13012017-0670-0

Dipali, U., \& Dumbre,. (2019). A Study to Assess the Knowledge and Compliance of Critical Care Nurses Regarding Ventilator Care Bundle in Prevention of Ventilator Associated Pneumonia. Medico Legal Update, 19(1), 176-178. Retrieved 4 April 2021 from:https://doi.org/10.37506/mlu. v19i1.908.

Arumugam, Y., Sivakumar, \& M.N., Balasubramanian, K. (2016). The impact of teaching on nurses' knowledge to VAP prevention bundle. Ind J Resp Care, 5(1), 6913.

Aferu ,B.(2016).Assessment of Knowledge and Practice of nurses working in ICU toward Prevention of ventilator associated Pneumonia at Selected governmental hospitals Addis Ababa, Ethiopia, Master Thesis in Adult Health Nursing, College of Health Sciences, Addis Ababa University,1-53.

Alcan, A.O., Giersbergen, M.Y., Yoltay, H.E., Geçkil, Ö.S., \&Solak, M. (2016). Turkish Nurses Awareness ofVentilator Bundle. JSM Allergy and Asthma, 1 (1), 1004, Retrieved from: https://www.jscimedcentral.com/A 1lergy/allergy-1-1004.pdf.

Ali, N.S. (2013). Critical Care Nurses' Knowledge and Compliance with Ventilator Associated Pneumonia Bundle at Cairo University Hospitals, Journal of Education and Practice, 4(15), 66-77 Retrieved from www.iiste.org/Journals/index.php/ JEP/article/viewFile/6821/6934. 
Al-Sayaghi, K.M. (2014). Prevention of ventilator-associated pneumonia. A knowledge survey among intensive care nurses in Yemen. Saudi Med J, 35(3), 26976,Retrieved from https://www.researchgate.net/.../26 0760483 Prevention of ventilato.

Subramanian, P., Choy, K. L., Gobal, S. V., Mansor, M., \& Ng, K. H. (2013). Impact of education on ventilator-associated pneumonia in the intensive care unit. Singapore medical journal, 54(5), 281-284.Retrived from:

https://doi.org/10.11622/smedj.201 3109.

Sobeih, H.S., Abd-EIsalam, S.N., \& Ahmed, R.Q. (2018). Ventilator Associated Pneumonia Bundle among Mechanically Ventilated Patient: Nurses' Perception. Egyptian Journal of Health Care, 9(2), 264-277. doi: 10.21608/ejhc.2018.20246.

Haza'a, A., Al-Qubati, F., Mohammed, M., Abdel-Aziz, M., \& Mehany, M. (2020). Effect of An Educational Program on Critical Care Nurses Performance regarding Emergency Care for Patients with Pulmonary Embolism. Assiut Scientific Nursing Journal, 8(20), 31-44. doi: 10.21608/asnj.2020.78956.

Kao, C., Chiang, H., Chen, C., Hung, C., Chen, Y., \& Su, L. et al. (2019). National bundle care program implementation to reduce ventilator-associated pneumonia in intensive care units in Taiwan. Journal Of Microbiology, Immunology And Infection, 52(4), 592-597. doi:
Walimanna Gamage, R. (2019). Audit on compliance of ventilator associated pneumonia care bundle in Intensive Care units in National Hospital Sri Lanka. Sri Lankan Journal Of Anaesthesiology, 27(1), 73. doi: 10.4038/slja.v27i1.8387

Branco, Aline, Lourençone, Emerson Matheus Silva, Monteiro, Ariane Baptista, Fonseca, Jaqueline Pettitembert, Blatt, Carine Raquel, \& Caregnato, Rita Catalina Aquino. (2020). Education to prevent ventilator-associated pneumonia in intensive care unit. Revista Brasileira de Enfermagem, 73(6), e20190477. Retrieved from: https://dx.doi.org/10.1590/00347167-2019-0477

Alsoda, M.F., Al-Shahat, M.M., Reda, S.M., Alsawah, A.Y., Abboud, M.A.,\& Elgendy, A.E.(2019). Implementation of ventilator bundle for prevention of ventilator-associated pneumonia in pediatric intensive care unit. J Med Sci Res, 2, 265-272

Álvarez Lerma, F., Sánchez García, M., Lorente, L., Gordo, F., Añón, J. M., Álvarez, J., Palomar, M., García, R., Arias, S., Vázquez-Calatayud, M., Jam, R., Sociedad Española de Medicina Intensiva, \& Sociedad Española de Enfermería Intensiva (2014). Guidelines for the prevention of ventilatorassociated pneumonia and their implementation. The Spanish "Zero-VAP" bundle. Medicina intensiva, 38(4),226-236.

Retrieved from: https://doi.org/10.1016/j.medin.20 13.12 .007 
Mogyoródi, B., Dunai, E., Gál, J., \& Iványi, Z. (2016). Ventilatorassociated pneumonia and the importance of education of ICU nurses on prevention - Preliminary results. Interventional medicine \& applied science, 8(4), 147-151. Retrieved from: https://doi.org/10.1556/1646.8.201 $\underline{6.4 .9}$

Oner Cengiz, H., \& Kanan, N. (2019). The effectiveness of training given to nurses for reducing ventilator-associated pneumonia in intensive care patients. Developments In Health Sciences, 2(2), 36:45. doi: 10.1556/2066.2.2019.006.

Akin Korhan, E., Hakverdioglu Yont, G., Parlar Kilic, S., Uzelli, D.(2014). Knowledge levels of intensive care nurses on prevention of ventilator-associated pneumonia. Nurs Crit Care. 19(1),26:33.

Maurya, S., Mishra, S.B., Azim, A., Baronia, A,K.,\& Gurjar, M.(2016). Ventilator-associated complications: a study to evaluate the effectiveness of a planned teaching program for intensive care unit staff nurses-an Indian experience. Am J Infect Control, 44(11), 1422:3.

Al-Sayaghi, K.M. (2014). Prevention of ventilator-associated pneumonia: a knowledge survey among intensive care nurses in Yemen. Saudi Med J, 35(3),269:76.

Jansson, M., Ala-Kokko, T., Ylipalosaari, P., Syrjälä, H.,\& Kyngäs, H.(2013). Critical care nurses' knowledge of, adherence to and barriers towards evidencebased guidelines for the prevention of ventilator-associated pneumonia - a survey study. Intensive Crit Care Nurs. 29(4), 216:27.

Dipanjali, R., Shivananda, P., \& Yashoda, S. (2021). Effectiveness of an Educational Intervention on Knowledge and Practice of Staff Nurses on Prevention of Ventilator Associated Pneumonia among Neonates in Neonatal Intensive Care Unit. International Journal Of Caring Sciences, 13(2), 1421:1430. doi: http://www.internationaljournalofc aringsciences.org/docs/68 $1 \mathrm{mam}$ antha original 13 2.pdf.

Meherali, S. M., Parpio, Y., Ali, T. S., \& Javed, F. (2011). Nurses' knowledge of evidence based guidelines for prevention of ventilator-associated pneumonia in critical care areas: a pre and post test design. Journal of Ayub Medical College, 23(1), 146- 149.

Al-Otaibi, H.M.(2019). Evaluation of critical care providers' knowledge in basic mechanical ventilation management: An opportunity for improvement. Saudi J Health Sci, 28, 162-6.

Retrieved from: https://www.saudijhealthsci. org/text.asp?2019/8/3/162/272439.

Fulbrook, P., Albarran, J.W., Baktoft, B.,\& Sidebottom B.(2012). A survey of European intensive care nurses' knowledge levels. Int J Nurs Stud, 49,191;200.

Bahgat, R. (2018). Effect of Educational Training Program about Chest Physiotherapy on Nurses' Performance and Clinical Outcomes for infants with Lower Respiratory Problems in Pediatric Intensive Care Unit, 8., 55:67. 
Baby, N. (2012). A study to assess the knowledge and practice of documentation techniques among staff nurses in selected hospitals, at davangere with a view to develop an information booklet. Rajiv Gandhi University of Health Sciences, Karnataka, India.

Ramya, K.,\& Neethu, J.(2013). Effectiveness of self-instructional module on nursing management of patients with chest tube drainage for staff nurses working in a selected hospital of Odisha. International $\mathrm{J}$ of Nursing Education, 5(2), 155:159.

Abdelazeem, E., Fashafsheh, I., \& Fadllalah, H. (2019). Effect of Training Program on Nurses Knowledge and Competence Regarding Endotracheal Tube and Tracheostomy Care in Mechanically Ventilated Patients. INTERNATIONAL
JOURNAL OF NURSING, 6(1). doi: 10.15640/ijn.v6n1a6.

Bedier, N., Abo EL-Ata, A.,\& Shehab. M (2020). Effect of Educational Program on Nurses' Practice Related to Care of Patients Undergoing Nasogastric Tube Feeding, 2:432.

Ghimire, S., \& Neupane, S. (2018). Knowledge regarding prevention of ventilator associated pneumonia among nurses in a tertiary hospital, Rupandehi, Nepal. Journal of Universal College of Medical Sciences, 6, 27:31.

Rakhi, M., \& Navita, R. (2020). Effectiveness of Structured Teaching Program on Knowledge and Practice Regarding Care Bundle on Prevention of Ventilator-Associated Pneumonia among Nurses. International Archives Of Nursing And Health Care, 6(4). doi: 10.23937/2469$5823 / 1510149$. 